Original Research Paper

\title{
K121Q Polymorphism of the ENPP1 Gene is Related to Acute Coronary Syndrome in Ukrainian Patients with Normal but not Enhanced Body Mass Index
}

\author{
${ }^{1}$ Inna A. Rozumenko, ${ }^{2}$ Victoria Y. Garbusova, \\ ${ }^{3}$ Yurij A. Ataman, ${ }^{4}$ Alexey V. Polonikov and ${ }^{1}$ Alexander V. Ataman \\ ${ }^{I}$ Department of Physiology, Pathophysiology and Medical Biology, Sumy State University, Sumy, Ukraine \\ ${ }^{2}$ Scientific Laboratory of Molecular Genetic Research, Sumy State University, Sumy, Ukraine \\ ${ }^{3}$ Department of Family Medicine with Propaedeutic of Internal Diseases and Endocrinology, \\ Sumy State University, Sumy, Ukraine \\ ${ }^{4}$ Department of Biology, Medical Genetics and Ecology, \\ Kursk State Medical University, Kursk, Russian Federation
}

Article history

Received: 05-11-2014

Revised: $13-11-2014$

Accepted: 08-01-2015

Corresponding Author:

Alexander V. Ataman

Department of Physiology,

Pathophysiology and Medical

Biology,

Sumy State University,

Sumy, Ukraine

Email: olex0101@gmail.com

\begin{abstract}
Ectonucleotide Pyrophosphatase Phosphodiesterase 1 (ENPP1) is a class II membrane glycoprotein with two unrelated properties: It can hydrolyze extracellular nucleotides and downregulate insulin receptor signaling. The present study was carried out to investigate whether common single-nucleotide polymorphism K121Q (rs1044498) of the ENPP1 gene is associated with Acute Coronary Syndrome (ACS) in the representatives of Ukrainian population and to assess if the risk depends on gender and Body Mass Index (BMI). A total 228 DNA samples (118 ACS patients and 110 control subjects) were genotyped for the polymorphism by PCR and restriction fragment length polymorphism method. No associations between the K121Q polymorphisms and ACS were found neither on the whole nor taking into account the gender. However, in the persons with $\mathrm{BMI}<25 \mathrm{~kg} / \mathrm{m}^{2}$ but not with overweight, genotypes with the minor allele (KQ + QQ) were significantly associated with ACS (OR 3.939, $95 \%$ CI 1.148-13.524, P = 0.029). Genotypes with minor allele can be a possible genetic risk factor for ACS in persons with BMI $<25 \mathrm{~kg} / \mathrm{m}^{2}$. It is likely that these genotypes affect ACS not by the traditional risk factors (overweight/obesity, insulin resistance and type 2 diabetes) but by direct or undirect influence on pathologic processes in the wall of the coronary vessels (atherosclerosis and arterial calcification).
\end{abstract}

Keywords: Ectonucleotide Pyrophosphatase Phosphodiesterase 1 (ENPP1), Acute Coronary Syndrome, Polymorphism, Body Mass Index

\section{Introduction}

Ectonucleotide Pyrophosphatase Phosphodiesterase 1 (ENPP1), also known as plasma cell membrane glycoprotein 1 (PC-1), belongs to a class II membrane glycoprotein which is widely expressed in many organs and tissues of the human and animal organisms (liver, skeletal muscle, heart, brain, kidney, lung, adipose tissue, etc.). Nowadays, the biological role of ENPP1 is not fully understood, but there are two groups of evidences concerning the ENNP1 significance in pathogenesis of some pathological processes and diseases.

The first group is about the ability of ENPP1 to influence insulin sensitivity by downregulating insulin receptor signaling (Dong et al., 2005). It was shown that over-expression of ENPP1 inhibits tyrosine kinase activity with subsequent diminishing insulin receptor autophosphorylation in various cells (Maddux and Goldfine, 2000). This property of ENPP1 is considered to be in association with insulin resistance and type 2 diabetes (Goldfine et al., 2007).

The second group of data is related to the processes of ectopic calcification. It is known that inorganic Pyrophosphate (PPi) is one of the most important inhibitor of soft tissues mineralization (Abedin et al., 2004; Shao et al., 2006). The main way of $\mathrm{PP}_{\mathrm{i}}$ generating is hydrolysis of extracellular nucleoside 
triphosphates, particular ATP, due to ENPP1 specific enzymatic activity (Johnson et al., 2005; Towler, 2005). When the activity of ENNP1 falls off the formation of $\mathrm{PP}_{\mathrm{i}}$ is disturbed and calcification of arteries may develop in many cases. The massive arterial calcification is the most impressive feature in genetically knocked out mice (ENPP1 -/-) and in humans who have defective ENNP1 gene (Johnson et al., 2005).

As a general rule, Acute Coronary Syndrome (ACS) is the consequence of atherosclerotic lesions appearing in arterial walls. It is well known that one of the main risk factors of atherosclerosis is type 2 diabetes which is frequently and tightly associated with insulin resistance. On the other hand, the coronary arteries calcification, as shown in many studies, is an adverse prognostic feature with regard to myocardial infarction in patients with atheromatous plaques (Lehto et al., 1996).

Taking into consideration the stated above, a relation of various Single-Nucleotide Polymorphisms (SNPs) of ENPP1 gene to ACS is of great interest. The most widely investigated ENPP1 SNP in genotype-phenotype association studies is the polymorphism K121Q. In this SNP located in exon 4, a lysine $(\mathrm{K})$ is substituted by a glutamine (Q) at codon 121 (Pizzuti et al., 1999).

There are some studies in which association of the ENPP1 K121Q polymorphism with early onset of coronary artery disease in Caucasians has been investigated (Endler et al., 2002; Bacci et al. 2005), but the data obtained in various ethnic groups remain controversial (Chen et al., 2006).

The aim of the present study was to perform a casecontrol study on representatives of the Ukrainian population in order to assess the possible association of the ENPP1 K121Q polymorphism with ACS in subjects of both genders who had normal and overweight levels of Body Mass Index (BMI).

\section{Materials and Methods}

\section{Subjects}

The study recruited 118 ACS patients $(78 \%$ men and $22 \%$ women) from 32 to 78 years of age (mean age $[ \pm \mathrm{SE}]$ $55.9 \pm 0.89)$ admitted to Sumy Clinical Hospital No.1.

Diagnosis of ACS was established on the basis of clinical, electrocardiography and biochemical examinations according to the recommendations of WHO experts and also according to recommendations of European and American cardiologic societies (Antman et al., 2004; Thygesen et al., 2007). Patients with hereditary and congenital diseases, severe metabolic pathologies including a severe form of diabetes mellitus, marked renal and liver failures, deficiencies of the haemostatic system, oncology and systemic pathologies, chronic heart failure of IIB-III stage, true cardiogenic shock were excluded from the study group.

The control group consisted of 110 clinically healthy individuals with the absence of cardio- and cerebrovascular pathologies, as confirmed by medical history, ECG and measurement of arterial pressure and biochemical data. The study had been previously approved by the Ethic Committee of the Medical Institute of Sumy State University. Appropriate informed consent was obtained from all patients and control subjects. The participants were unrelated Ukrainian people from the northeastern region of Ukraine.

The subjects of both groups were divided into subgroups by gender and by BMI $\left(B M I<25 \mathrm{~kg} / \mathrm{m}^{2}\right.$ and $\geq 25 \mathrm{~kg} / \mathrm{m}^{2}$ ).

Blood sampling for genotyping was performed under sterile conditions into $2.7 \mathrm{ml}$ tubes (SMonovette [Sarstedt, Germany]) containing EDTA potassium salt as an anticoagulant, samples were frozen and stored at $-20^{\circ} \mathrm{C}$.

\section{Genotyping of SNP}

DNA for genotyping was extracted from the venous blood using commercially available kits (Isogene Lab Ltd, Russia) according to the manufacturer's protocol. To identify ENPPI K121Q polymorphism (rs1044498) the Polymerase Chain Reaction (PCR) with subsequent Restriction Fragment Length Polymorphism (RFLP) analysis was performed. Specific region of the ENPPI gene was amplified using a pair of specific primers: Upstream (sense)-5' CTGTGTTCACTTTGGACATGTTG 3' and downstream (antisense)-5' GACGCTGGAagAtACCAGGCTG 3'. Primers were provided by Metabion (Germany). PCR was performed for 33 cycles in a $25 \mu \mathrm{L}$ volume containing 50-100 ng of DNA, $5 \mu \mathrm{L} 5 \mathrm{X}$ PCR-buffer, $1.5 \mathrm{mM}$ magnesium sulfate, $200 \mu \mathrm{M}$ of each dNTP, $15 \mathrm{pM}$ of each primer and $0.75 \mathrm{U}$ of Taq DNA polymerase (Thermo Scientific, USA). PCR was carried out in a termocycler GeneAmp PCR System 2700 (Applied Biosystems, USA). Six microlitres $(6 \mu \mathrm{L})$ of the PCR products $(238 \mathrm{bp})$ were subjected to digestion with $5 \mathrm{U}$ Eco47I (AvaII) (Thermo Scientific, USA) and incubated at $37^{\circ} \mathrm{C}$ for $18 \mathrm{~h}$. In case of the presence of cytosine at the position 48213 of the exon 4, Eco47I restriction enzyme produces two fragments of 148 and $90 \mathrm{bp}$ in length. Substitution of cytosine for adenine prevents restriction and the amplified fragment of the exon 4 (238bp) can not be cleaved. The restriction fragments were separated by electrophoresis and analysed on an ethidium bromide-stained $2.5 \%$ agarose gel visualized using ultraviolet transillumination. 


\section{Statistical Analysis}

The normal distribution and homogeneity of variances were tested before further statistical analyses. The comparison of variables between the groups of genotypes was performed using two-tailed Student's $t$ test. The $\chi 2$-test was used for comparison of the allele and genotype frequencies between different studied groups and subgroups. Odds ratio was evaluated by using the logistic regression method. The differences were considered statistically significant with a P-value $<0.05$. All statistical analyses were performed using the Statistical Package for Social Science program (SPSS for Windows, version 17.0, SPSS Inc., Chicago, IL).

\section{Results}

The clinical characteristics of 118 patients with ACS and 140 healthy controls are summarized in Table 1. No differences between the groups were noted with respect to sex, age or body mass index. Atherogenic risk factors (including cigarette smoking, hypertension, total cholesterol and glucose concentration) were significantly more prevalent in the ACS patient group.

In Figure 1 the results of RFLP analysis of ENPP1 $\mathrm{K} 121 \mathrm{Q}$ polymorphism are demonstrated. In both groups we studied, the genotype distributions of the ENPP1 K121Q polymorphism were in Hardy-Weinberg equilibrium. The minor allele frequencies of SNP were not significantly different between the ACS (0.18) and control (0.12) groups. These frequencies were comparable with other studies using the same SNP in populations of European descent (Bottcher et al., 2006; Morandi et al., 2009).

For genotype case-control analysis we took into consideration two subgroups (KK and KQ+QQ) since there were very few minor allele homozygotes QQ both in ACS (3 individuals) and in control ( 0 subjects) groups. As shown in Table 2, major allele homozygotes $\mathrm{KK}$ and carriers of minor allele KQ+QQ were detected in 66.9 and $33.1 \%$ of the ACS group, respectively (control group: 75.5 and 24.5\%). The differences in the distribution of allelic variants between the ACS and control groups were not statisticaly significant. Such conclusion was true when subjects of both groups were divided into subgroups by gender but not by BMI.

Using logistic regression analysis (Table 3 ), it was estimated that in persons with BMI $<25 \mathrm{~kg} / \mathrm{m}^{2}$, carrying of minor allele (KQ+QQ) was significantly associated with ACS $(\mathrm{OR}=3.939$; 95\% CI, 1.148-13.524, $\mathrm{P}=$ 0.029). The same could not be said about individuals with overweight $\left(\mathrm{BMI} \geq 25 \mathrm{~kg} / \mathrm{m}^{2}\right)$.

\section{Discussion}

In the present study, we explored associations between genetic variation in the ENPPI gene and ACS risk. Analyzing the SNP of this gene we found that $\mathrm{K} 121 \mathrm{Q}$ polymorphism in exon 4 was associated with ACS in the representatives of Ukrainian population only with normal $\left(\mathrm{BMI}<25 \mathrm{~kg} / \mathrm{m}^{2}\right)$, but not enhanced weight. The risk of ACS in not-overweight persons who were carriers of minor Q-allele was 3.9 fold greater as compared with main K-allele homozygotes.

A functional missense DNA polymorphism in exon 4 causes an amino acid change from lysine to glutamine at codon 121 (K121Q) (Pizzuti et al., 1999). Studies in vitro have shown that the $\mathrm{Q}$ variant of ENPP1 binds insulin receptors more strongly than the $\mathrm{K}$ variant and reduces insulin receptor autophosphorylation (Costanzo et al., 2001). It is therefore a stronger inhibitor of insulin signaling.

In this connection wide range of case-control studies were conducted to asses the possible association between the ENPP1 gene K121Q polymorphism and (1) insulin resistance, (2) Type 2 Diabetes Mellitus (T2DM) and (3) overweight/obesity in many Caucasian and not-Caucasian populations (Goldfine et al., 2007). Although the published results are rather contradictory, it can be concluded that in many cases there are positive associations between $Q$ variant of ENPP1 gene and aforementioned pathological conditions.

All of these conditions are considered to be tightly pathogenetic connected with atherosclerotic process which is the main cause of ACS. This should be taken into account when studying relationship of the ENPP1 gene SNPs to cardiovascular diseases.

There is evidence suggesting that the $\mathrm{Q}$ allele is associated with an increased risk of earlier onset of myocardial infarction (Endler et al., 2002; Bacci et al., 2005). This association may be secondary to the effect of the $\mathrm{Q}$ allele on insulin resistance, T2DM and overweight/obesity which all predispose to atherosclerosis (Fig. 2).

In our study, we showed that ENPP1 gene K121Q polymorphism is related to ACS only in nondiabetic patients with normal weight and fasting glucose levels. It suggested the possible impact of this SNP on ACS not only by overweight/obesity and T2DM, but also by processes witch cause coronary insufficiency, i.e., atherosclerosis and arterial calcification.

The influence of ENPP1 overexpression on arterial wall may be in two different ways (Fig. 3). On the one hand, downregulating insulin receptor signaling by ENPP1 leads to insulin resistance and contribute to atherosclerotic lesions development. On the other hand, enhanced enzymatic activity of ENPP1 should increase the formation of $\mathrm{PP}_{\mathrm{i}}$ which is one of the most potent anticalcinogenic factor that can prevent arterial wall calcification.

It is yet not known how $\mathrm{K} 121 \mathrm{Q}$ polymorphism impacts on enzymatic feature of ENPP1. This is a question that needs to be under further investigation. 


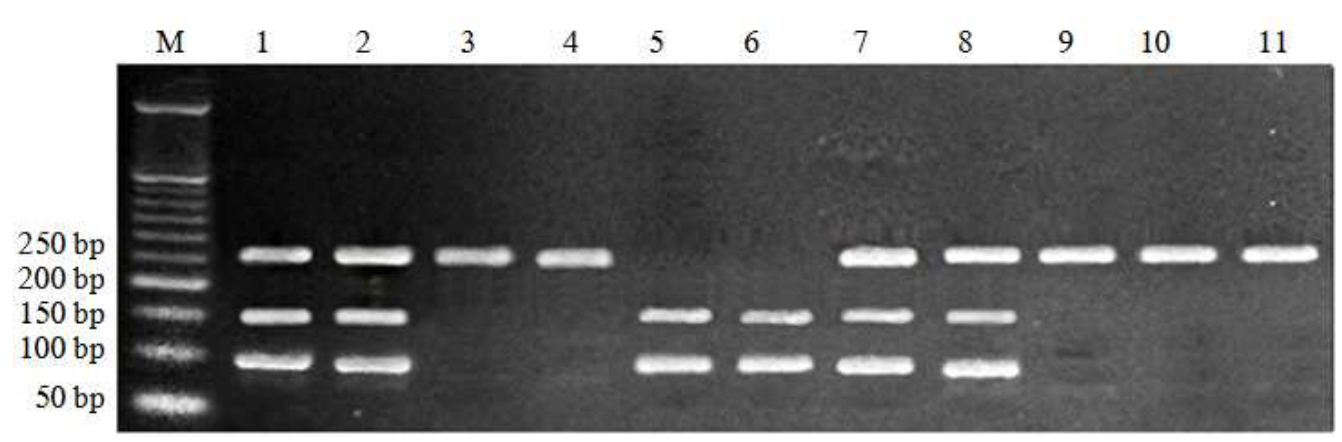

Fig 1. Analysis of ENPP1 K121Q polymorphism. Results of restriction fragment electrophoresis of polymerase chain reaction amplification products. M-molecular marker (bp-base pairs), lanes 3, 4, 9, 10, 11 (KK genotype); lanes 1, 2, 7, 8 (KQ genotype); lanes 5, 6-(QQ genotype)

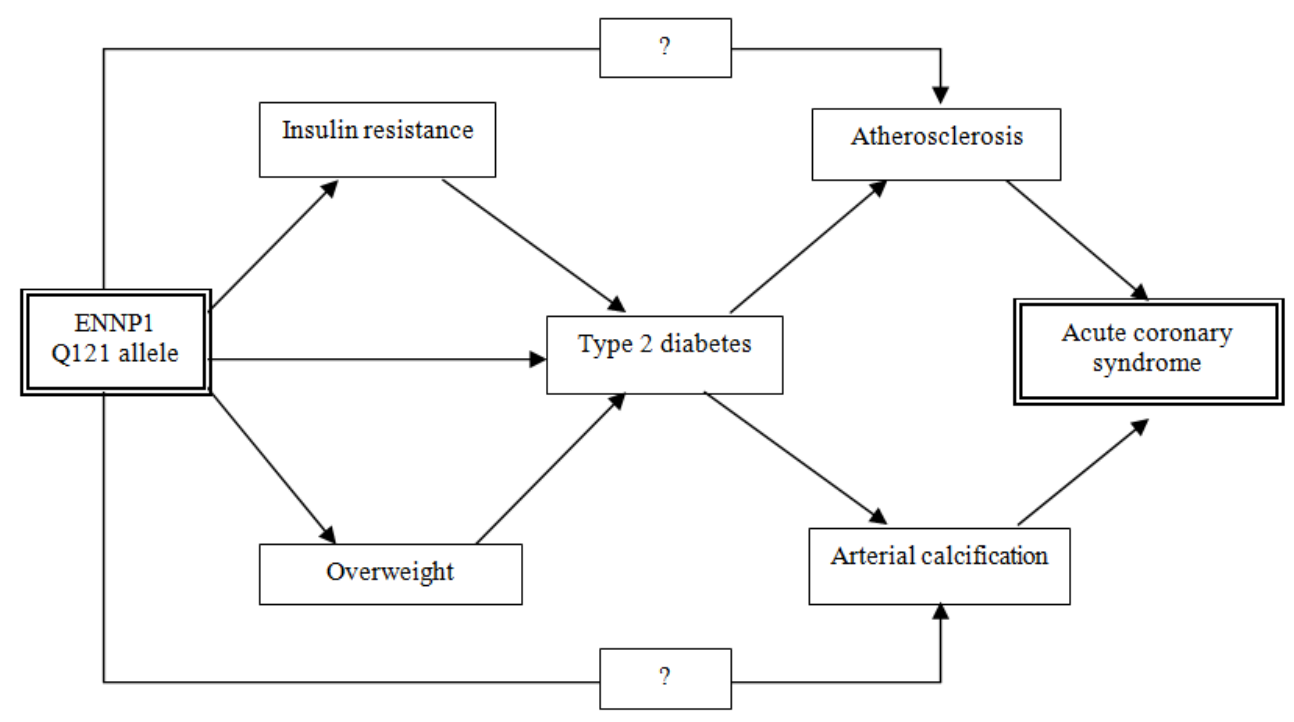

Fig. 2. Possible ways of ENNP1 Q121 allele impact on the pathogenesis of acute coronary syndrome

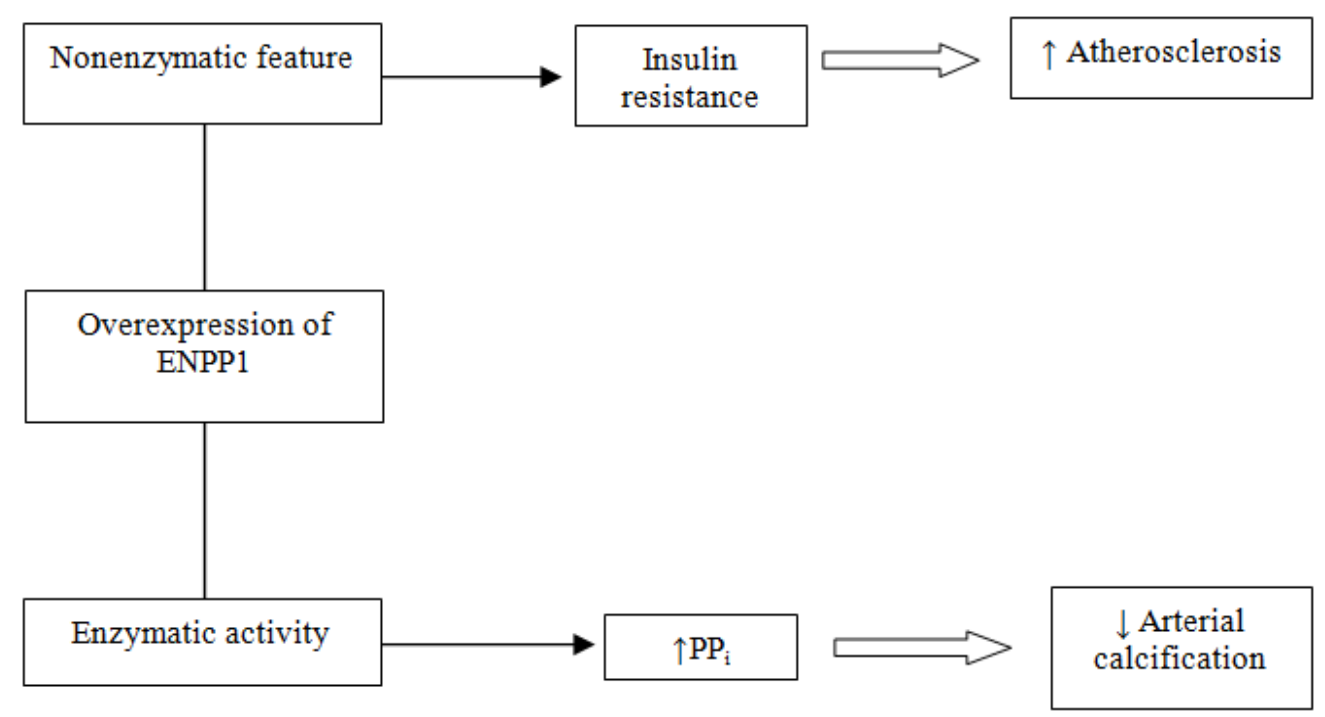

Fig. 3. Two possible opposite effects of ENPP1 overexpression on the wall of coronary arteries 
Table 1. Clinical parameters of Acute Coronary Syndrome (ACS) and healthy (control) subjects

\begin{tabular}{llll} 
& Subjects & & \\
Parameters & - & & \\
Sex, male/female, $\mathrm{n} / \mathrm{n}$ & ACS $(\mathrm{n}=118)$ & Control $(\mathrm{n}=110)$ & $0.221^{*}$ \\
Age, years & $92 / 26$ & $78 / 32$ & 0.105 \\
Current smokers, $\mathrm{n}(\%)$ & $55.9 \pm 0.89$ & $54.0 \pm 0.74$ & $0.002^{*}$ \\
BMI, $\mathrm{kg} / \mathrm{m}^{2}$ & $54(45.8)$ & $29(26.4)$ & 0.022 \\
SBP, $\mathrm{mm} \mathrm{Hg}$ & $28.5 \pm 0.41$ & $27.1 \pm 0.41$ & $<0.001$ \\
DBP $\mathrm{mm} \mathrm{Hg}$ & $140.8 \pm 1.7$ & $124.6 \pm 1.0$ & $<0.001$ \\
TC, $\mathrm{mmol} / \mathrm{L}$ & $89.5 \pm 0.9$ & $80.2 \pm 0.7$ & 0.004 \\
HDL-C, $\mathrm{mmol} / \mathrm{L}$ & $6.4 \pm 0.13$ & $5.6 \pm 0.25$ & 0.012 \\
Fasting glucose, $\mathrm{mmol} / \mathrm{L}$ & $1.0 \pm 0.12$ & $1.4 \pm 0.10$ & $<0.001$ \\
\hline
\end{tabular}

Table 2. Genotypes of ENPP1 K121Q polymorphism in patients with Acute Coronary Syndrome (ACS) and control subjects

\begin{tabular}{|c|c|c|c|c|c|c|}
\hline \multirow[b]{2}{*}{ Genotype } & \multicolumn{2}{|l|}{ Total } & \multicolumn{2}{|l|}{ Men } & \multicolumn{2}{|l|}{ Women } \\
\hline & $\begin{array}{l}\text { ACS group } \\
(\mathrm{n}=118)\end{array}$ & $\begin{array}{l}\text { Control group } \\
(\mathrm{n}=110)\end{array}$ & $\begin{array}{l}\text { ACS group } \\
(\mathrm{n}=92)\end{array}$ & $\begin{array}{l}\text { Control group } \\
(\mathrm{n}=78)\end{array}$ & $\begin{array}{l}\text { ACS group } \\
(\mathrm{n}=26)\end{array}$ & $\begin{array}{l}\text { Control group } \\
(\mathrm{n}=32)\end{array}$ \\
\hline $\mathrm{KK}$ & $79(66.9)$ & $83(75.5)$ & $62(67.4)$ & $58(74.4)$ & $17(65.4)$ & $25(78.1)$ \\
\hline KQ+QQ & $39(33.1)$ & $27(24.5)$ & $30(32.6)$ & $20(25.6)$ & $9(34.6)$ & 7 (21.9) \\
\hline OR $(95 \% \mathrm{CI})$ & \multicolumn{2}{|c|}{$1.518(0.850-2.709)$} & \multicolumn{2}{|c|}{$1.891(0.590-6.056)$} & \multicolumn{2}{|c|}{$1.403(0.718-2.741)$} \\
\hline P-value & \multicolumn{2}{|c|}{0.158} & \multicolumn{2}{|c|}{0.284} & \multicolumn{2}{|c|}{0.321} \\
\hline
\end{tabular}

Table 3. Genotypes of ENPP1 K121Q polymorphism in Acute Coronary Syndrome (ACS) and control subjects with normal $(<25$ $\left.\mathrm{kg} / \mathrm{m}^{2}\right)$ and enhanced $\left(\geq 25 \mathrm{~kg} / \mathrm{m}^{2}\right)$ Body Mass Index (BMI)

\begin{tabular}{llll}
\hline & BMI $<25$ & BMI $\geq 25$ \\
Genotype & ACS & Control \\
$(\mathrm{n}=32)$ & $21)$ & $\begin{array}{l}\text { ACS } \\
(\mathrm{n}=97)\end{array}$ \\
\hline KK & $11(52.4)$ & $26(81.3)$ & $68(70.1)$ \\
KK+KQ & $10(47.6)$ & $6(18.7)$ & $29(29.9)$ \\
OR $(95 \% \mathrm{CI})$ & $3.939(1.148-13.524)$ & & $1.158(0.597-2.246)$ \\
P-value & 0.029 & & 0.665 \\
\hline
\end{tabular}

\section{Conclusion}

In the present study, genotypes with minor allele (KQ+QQ) for ENPP1 K121Q polymorphism were observed as a possible genetic risk factor for ACS only in persons with BMI $<25 \mathrm{~kg} / \mathrm{m}^{2}$. It is more likely that these genotypes affect ACS not through the traditional risk factors (overweight/obesity, insulin resistance and type 2 diabetes) but by direct or undirect influence on pathologic processes in the wall of the coronary vessels (atherosclerosis and arterial calcification).

\section{Acknowledgement}

We thank all the consultants for their invaluable help during sample collection. We appreciate the participation of all the subjects who volunteered for this study. The authors declare that they have no conflict of interest.

\section{Funding Information}

The study was a part of scientific project "Association of ectopic calcification genes polymorphisms with widespread cardiovascular diseases and their complications" supported by the Ministry of Education and Science of Ukraine, 2013-2014 (No 0113U000132).

\section{Author's Contributions}

Inna A. Rozumenko: Wrote the manuscript, performed genotyping

Victoria Y. Garbusova: Performed genotyping and biostatistics

Yurij A. Ataman: Performed the clinical research

Alexey V. Polonikov: Coordinated the data-analysis, contributed to writing manuscript

Alexander V. Ataman: Designed the research plan and organized the study 


\section{Ethics}

The study has been approved by the Ethic Committee of the Medical Institute of Sumy State University.

\section{References}

Abedin, M., Y. Tintut and L.L. Demer, 2004. Vascular calcification. Mechanisms and clinical ramifications. Arteriosclerosis, Thrombosis Vascul. Biol., 24: 1161-1170. DOI: 10.1161/01.ATV.0000133194.94939.42

Antman, E.M., D.T. Anbe, P.W. and Armstrong, M.D.R.E. Bates and M.D.L.A. Green et al., 2004. ACC/AHA guidelines for the management of patients with ST-elevation myocardial infarction. J. Am. College Cardiol., 44: 671-719. DOI: 10.1016/j.jacc.2004.07.002

Bacci, S., O. Ludovico and S. Prudente, Y.Y. Zhang and R.D. Paola et al., 2005. The K121Q polymorphism of the ENPP1/PC-1 gene is associated with insulin resistance/atherogenic phenotypes, including earlier onset of type 2 diabetes and myocardial infarction. Diabetes, 54: 3021-3025. DOI: 10.2337/diabetes.54.10.3021

Bottcher, Y., A. Korner and T. Reinehr, B. Enigk and W. Kiess et al., 2006. ENPP1 variants and haplotypes predispose to early onset obesity and impaired glucose and insulin metabolism in German obese children. J. Clin. Endocrinol. Metabol., 91: 4948-4952. DOI: $10.1210 /$ jc.2006-0540

Chen, M.P., F.M. Chung and D.M. Chang, J.C.R. Tsai and H.F. Huang et al., 2006. ENPP1 K121Q polymorphism is not related to type 2 diabetes mellitus, features of metabolic syndrome and diabetic cardiovascular complications in a Chinese population. Rev. Diabetic Stud., 3: 21-30.

DOI: 10.1900/RDS.2006.3.21

Costanzo, B.V., V. Trischitta and R. Di Paola, D. Spampinato and A. Pizzuti et al., 2001. The Q allele variant (GLN121) of membrane glycoprotein PC-1 interacts with the insulin receptor and inhibits insulin signaling more effectively than the common Kallele variant (LYS121). Diabetes, 50: 831-836. DOI: $10.2337 /$ diabetes.50.4.831

Dong, H., B.A. Maddux and J. Altomonte, M. Meseck and D. Accili et al., 2005. Increased hepatic levels of the insulin receptor inhibitor, PC$1 / \mathrm{NPP} 1$, induce insulin resistance and glucose intolerance. Diabetes, 54: 367-372.

DOI: $10.2337 /$ diabetes.54.2.367
Endler, G., C. Mannhalter and H. Sunder-Plassmann, M. Schillinger and A. Klimesch et al., 2002. The $\mathrm{K} 121 \mathrm{Q}$ polymorphism in the plasma cell membrane glycoprotein 1 gene predisposes to early myocardial infarction. J. Molecul. Med., 80: 791-795. DOI: 10.1007/s00109-002-0385-8

Goldfine, I.D., B.A. Maddux and J.F. Youngren, G. Reaven and D. Accili et al., 2007. The role of membrane glycoprotein plasma cell antigen 1/ectonucleotide pyrophosphatase phosphodiesterase 1 in the pathogenesis of insulin resistance and related abnormalities. Endocrine Rev., 29: 62-75. DOI: 10.1210/er.2007-0004

Johnson, K., M. Polewski, D. van Etten and R. Terkeltaub, 2005. Chondrogenesis mediated by PPi depletion promotes spontaneous aortic calcification in NPP1-/- mice. Arterioscl., Thrombosis Vascul. Biol., 25: 686-691. DOI: 10.1161/01.ATV.0000154774.71187.f0

Lehto, S., L. Niskanen, M. Suhonen, T. Rönnemaa and M. Laasko, 1996. Medial artery calcification. A neglected harbinger of cardiovascular complications in non-insulin-dependent diabetes mellitus. Arterioscl., Thrombosis, Vascul. Biol., 16: 978-983. DOI: 10.1161/01.ATV.16.8.978

Maddux, B.A. and I.D. Goldfine, 2000. Membrane glycoprotein PC-1 inhibition of insulin receptor function occurs via direct interaction with the receptor alpha-subunit. Diabetes, 49: 13-19.

DOI: $10.2337 /$ diabetes.49.1.13

Morandi, A., L. Pinelli and A. Petrone, V. Vatin and R. Buzzetti et al., 2009. The Q121 variant of ENPP1 may protect from childhood overweight/obesity in the Italian population. Obesity, 17: 202-206. DOI: $10.1038 /$ oby.2008.470

Pizzuti, A., L. Frittitta and A. Argiolas, R. Baratta and I.D. Goldfine et al., 1999. A polymorphism (K121Q) of the human glycoprotein PC-1 gene coding region is strongly associated with insulin resistance. Diabetes, 48: 1881-1884. DOI: $10.2337 /$ diabetes.48.9.1881

Shao, J.S., J. Cai and D.A. Towler, 2006. Molecular mechanisms of vascular calcification: Lessons learned from the aorta. Arterioscl., Thrombosis, Vascul. Biol., 26: 1423-1430.

DOI: 10.1161/01.ATV.0000220441.42041.20

Thygesen, K., J. Alpert and H. White, 2007. Universal definition of myocardial infarction. Eur. Heart J., 28: $2525-2538$.

Towler, D.A., 2005. Inorganic pyrophosphate. A paracrine regulator of vascular calcification and smooth muscle phenotype. Arterioscl., Thrombosis Vascul. Biol., 25: 651-654.

DOI: 10.1161/01.ATV.0000158943.79580.9d 